\title{
GCU
}

Glasgow Caledonian

University

University for the Common Good

\section{An improved statistical time series method for voltage violation quantification in residential distribution network with small wind turbines and battery electric vehicles}

Long, Chao; Hepburn, Donald; Farrag (Emad), Mohamed Emad; Zhou, Chengke

Published in:

International Journal of Smart Grid and Clean Energy

DOI:

10.12720/sgce.3.1.29-36

Publication date:

2014

Document Version

Publisher's PDF, also known as Version of record

Link to publication in ResearchOnline

Citation for published version (Harvard):

Long, C, Hepburn, D, Farrag (Emad), ME \& Zhou, C 2014, 'An improved statistical time series method for voltage violation quantification in residential distribution network with small wind turbines and battery electric vehicles', International Journal of Smart Grid and Clean Energy, vol. 3, no. 1, 3, pp. 29-36.

https://doi.org/10.12720/sgce.3.1.29-36

\section{General rights}

Copyright and moral rights for the publications made accessible in the public portal are retained by the authors and/or other copyright owners and it is a condition of accessing publications that users recognise and abide by the legal requirements associated with these rights.

Take down policy

If you believe that this document breaches copyright please view our takedown policy at https://edshare.gcu.ac.uk/id/eprint/5179 for details

of how to contact us. 


\title{
An improved statistical time series method for voltage violation quantification in residential distribution network with small wind turbines and battery electric vehicles
}

\author{
Chao Long", Donald M. Hepburn, Mohamed E.A. Farrag, Chengke Zhou \\ School of Engineering and Built Environment, Glasgow Caledonian University, Cowcaddens Road, UK. G4 OBA
}

\begin{abstract}
The probabilistic power flow method has previously been used for voltage violation quantification in residential distribution networks (RDNs) with small wind turbines (SWTs) and battery electric vehicles (BEVs). An improved statistical time series approach is developed in this paper to account for factors which impact on system conformity. In addition to consideration of variations in wind speed over time and BEV charging, using a statistical time series approach, the novel method takes into account variations in system load caused by the randomness of load switching by customers. The method has been applied to a generic UK distribution network. Results show that the proposed method provides a closer indication of daily probability distribution of voltage violations in a RDN, based on physical data, than the previous statistical time series approach.
\end{abstract}

Keywords: Battery electric vehicles, probability distribution, small wind turbines, time series, voltage violation

\section{Introduction}

Increasing penetration of electric vehicles (EVs) and distributed power generating equipment, such as small wind turbines (SWTs), has been evident in residential distribution networks (RDNs) [1], [2]. The impact of the changes resulting from additional demand and generation capability will affect operation of RDNs. Voltage violation tends to be one of the dominant constraints or critical factors that limit a distribution network's ability to incorporate SWTs and EVs [3]-[5]. Due to the intermittent characteristics of wind speed, the resultant time-variations in SWT output will change either, or both, magnitude and direction of network power flow. The randomness of EV charging events, through plugging into a RDN, will impact on power required from the RDN grid connection point. The changes in power flowing through the cables as a result of localized power generation and battery charging will impact on voltage drop through the system. The lack of predictability of the power generation and charging load requirements introduce difficulties in evaluating voltage violation in RDNs.

According to Engineering Recommendation G83/1, a distributed generation (DG) unit rated up to 16 A per phase can be connected to distribution networks [6]. In this paper SWTs in RDNs refer to singlephase wind turbines of this type, i.e. at $230 \mathrm{~V}$ a.c. they are rated less than $3.68 \mathrm{kVA}$. In relation to gridconnected EVs, there are two primary types: battery EV (BEV) and plug-in hybrid EV (PHEV). For simplicity, this paper only considers BEVs.

In the $\mathrm{UK}$, for systems above $50 \mathrm{~V}$ and below $1 \mathrm{kV}$, unless otherwise agreed, voltage variations between $\pm 10 \%$ of nominal voltage are permitted. For the purposes of this paper when the voltage at any node of a distribution network breaches the statutory limits it is considered a voltage violation.

Due to the inherent variability in system loads and in SWT outputs, the application of a time series approach to power flow analysis can be advantageous [7]-[11]. The time series analysis is a method

\footnotetext{
* Manuscript received May 16, 2013; revised August 6, 2013.

Corresponding author Tel.: +44(0)141 331 3453; E-mail address: chao.long@ gcu.ac.uk.
} 
applied to execute power flow analyses in a set of time series with specific values of system load and generation for each time step [7]. For detailed analysis, a time window with a period of a day or a week is usually utilized. However, the specific time windows cannot provide a global picture of voltage violation of the network under study. Although several studies have executed power flow analysis by using the extreme conditions of minimum load and maximum DG output, e.g. [12] and [13], they failed to take into account the likelihood of the violation occurring. Therefore, it has been considered worthwhile to approach the voltage violation problem in a statistical manner.

The probabilistic power flow (PPF) method has been employed for voltage variation study of a distribution network in many previous works [14]-[18]. The PPF calculation can be divided into a numerical method, e.g. Monte Carlo simulation, and an analytical method. Using the Monte Carlo technique is computationally intense, a large number of simulations, e.g. 20,000, are required. To reduce the computational burden, the PPF calculation was performed with statistical distribution of loads and generations in [14]-[16]. A statistical model of wind generation was developed in [14], but the variation in load was not considered. In [15] and [16], the statistical distributions of wind generation in winter and summer were integrated to the network with winter and summer loads, respectively, but the timevariations in loads and wind speeds were not considered. A statistical time series method was developed in [18], and the time variations in wind speeds and BEV charging were considered. At different times of day, the wind speeds were discretized and considered in a statistical manner, a deterministic value of load following a typical daily load profile was assumed for each time instant, but the randomness of load switching by customs was not considered.

To develop the approaches previously taken, in addition to the consideration of time-variations in wind speeds and BEV charging in statistical time series approach, the improved method models the variations in system load caused by the randomness of load switching by customers. As a result, simulations using the proposed method are shown to provide results of probability distribution of voltage violations in a RDN which are closer to results based on physical data than using the statistical time series approach.

\section{Modeling}

In RDNs containing SWTs and BEVs, node voltages related to active and reactive power flow in branches can be obtained via steady-state power flow analysis. Power flow in RDNs depends on the instantaneous injection of electrical power from SWTs and the sum of individual electrical demands from customers. SWT generator outputs and loads should be modeled prior to the power flow analysis.

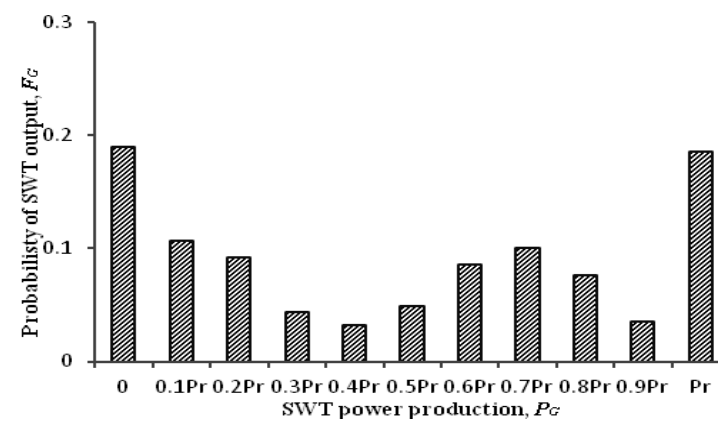

Fig. 1. The probabilistic distribution of SWT output, $M=10$.

\subsection{Statistical SWT model}

Due to the intermittent characteristic of wind speed, SWT output is neither continuous nor stable. To calculate the likely power output from a given SWT it is necessary to understand the wind behavior in the location under consideration. The Weibull distribution function is widely used to statistically describe the wind speed distribution, e.g. [14], [19]. The statistical SWT model used in [18] is employed in this work. All wind speed data at a specific instant is differentiated into discrete values. With a given SWT power 
output curve, discrete SWT output and the corresponding probabilities of the SWT output can be obtained from (1):

$$
F_{G}=\left\{P_{G}(i), F_{G}(i): i=0,1,2, \ldots, M\right\}
$$

where power $P_{G}(i)$ has $M+1$ states at which the probability of $P_{G}(i)$ is calculated from the discrete wind speeds and the SWT power output curve and $F_{G}(i)$ is the probability of SWT output for the $i$ th SWT output value, as shown in Fig. $1 M$ has been taken as 10 .

\subsection{Statistical model of load profile}

In [18], a typical residential load profile was extracted based on historical UK load data. At a specific time instant, a deterministic load value following the typical load profile was taken, so that the voltage violation under typical load conditions can be obtained. However, the load at a time instant varies in a random manner due to the variability of load switching by customers. In this paper the load has been considered as a random variable. Load data at the same time instant of day have been differentiated into discrete values. The probability distribution of the discrete values can be expressed by equation (2) In [18], a typical residential load profile was extracted based on historical UK load data. At a specific time instant, a deterministic load value following the typical load profile was taken, so that the voltage violation under typical load conditions can be obtained. However, the load at a time instant varies in a random manner due to the variability of load switching by customers. In this paper the load has been considered as a random variable. Load data at the same time instant of day have been differentiated into discrete values. The probability distribution of the discrete values can be expressed by equation (2) In [18], a typical residential load profile was extracted based on historical UK load data. At a specific time instant, a deterministic load value following the typical load profile was taken, so that the voltage violation under typical load conditions can be obtained. However, the load at a time instant varies in a random manner due to the variability of load switching by customers. In this paper the load has been considered as a random variable. Load data at the same time instant of day have been differentiated into discrete values. The probability distribution of the discrete values can be expressed by equation (2):

$$
F_{L}=\left\{L(l), F_{L}(l): l=1,2, \ldots, W\right\}
$$

where $L(l)$ is the discrete value of the load, at a time instant, and $W$ is the number of the discrete values. $F_{L}(l)$ is the corresponding probability of load at discrete value $L(l)$.

\subsection{Probabilistic model of BEV charging load}

The BEV charging load model presented in [2] is adopted in this paper. The load due to BEV charging is determined by several factors: the battery type, the initial state-of-charge (SOC) (residual capacity since last charge) and the start time of BEV charging. The lithium-ion battery selected for this work is based on that used in the Nissan Altra; it has recently undergone a rapid improvement in performance characteristics and an increase in popularity [20]. The power demand and related battery SOC profile of the battery has been taken from [21]. The power demand during a battery charging process is scattered with values $P_{E}(j)$ taken in constant time intervals from the curve of a battery charging profile. The corresponding power levels of BEV charging load are expressed using equation (3):

$$
P_{E}(j)=\frac{P_{E}((j-1) \Delta t)+P_{E}(j \Delta t)}{2}, j=1,2, \ldots, n_{c}
$$

where $P_{E}$ is the function of a battery charging power demand profile, $n_{c}$ is the number of intervals in the profile, $\Delta t$ is the constant time interval.

Using discretized values of the BEV charging load, following the method in [2] and [18], the probability distribution of the discrete values can be expressed by (4):

$$
\Phi=\left\{P_{E}(j), \Phi\left(P_{E}(j)\right): j=1,2, \ldots, n_{c}\right\}
$$


where $P_{E}(j)$ is the discrete value of BEV power demand, $n_{c}$ is the number of the discrete values, and $\Phi\left(P_{E}(j)\right)$ is the corresponding probability of power demand at value $P_{E}(j)$.

Based on the statistical analysis in [2], the probability distribution function ( $p d f)$ of the initial SOC was obtained. From this data, the initial SOC of private BEVs has a mean of $72 \%$ after one day's travel and $44 \%$ after two-day's travel. Thus, BEVs in a residential area are assumed to be charged every other day on average. The time at which a BEV is plugged in for charging depends on resident's choice: three domestic BEV charging schemes were employed in [2], uncontrolled charging, off-peak charging and smart charging. For simplicity, this paper assumes uncontrolled domestic charging, and that the charging start time is uniformly distributed over four hours (from 18:00 to 21:59). Therefore BEV charging will only impact on loading of a RDN between 18:00 and 3:00 the next day.

\section{Formulation}

The voltage at each node of a RDN must be maintained within the rated range:

$$
V_{x \min } \leq V_{x} \leq V_{x \max } \quad x \in N
$$

where $V_{x}$ is the voltage at the $x$ th node, and $V_{x \min }$ and $V_{x \max }$ are the minimum and maximum allowable voltage levels.

\subsection{Calculation of probability of voltage violation}

For simplicity two assumptions are made: firstly, the wind speed at any given time is the same for all SWTs in the small-scale area of the RDN; secondly, all BEV batteries in the network have an identical charging characteristic.

During the times when there are no BEV charging loads in the RDNs, voltages of the network depend on the original network load and SWT output. For SWT power output, assume $P(i)<P(i+1), 0 \leq i \leq M-1$; regarding load data, assume $L(l)<L(l+1), 0 \leq l \leq W-1$. At a time instant, when $i=\tau_{1}$ and $l=\tau_{2}\left(\tau_{1}, \tau_{2} \in N\right)$, voltage violation occurs, the probability of voltage violation in a RDN with SWTs, $P_{1}$, can be then expressed by (6):

$$
P_{1}=\left\{\begin{array}{l}
\sum_{l=\tau_{2}}^{W} \sum_{i=0}^{\tau_{1}} F_{G}(i) \cdot F_{L}(l) \text { when } V_{x}<V_{x \min } \\
\sum_{l=0}^{W} \sum_{i=\tau_{1}}^{M} F_{G}(i) \cdot F_{L}(l) \text { when } V_{x}>V_{x \max }
\end{array}\right.
$$

where $\tau_{1}, \tau_{2} \in N, 0 \leq \tau_{1} \leq M, 0 \leq \tau_{2} \leq W, x \in N$.

During periods when BEVs are connected to a RDN, the total load of the RDN would be the sum of the network loads and the BEV charging loads. The load distribution can be expressed by (7):

$$
\varphi=\left\{L_{R D N}(l j), \varphi_{l j}: l=1,2, \ldots, W, j=1,2, \ldots, n_{c}\right\}
$$

where $L_{R D N}(l j)=L(l)+P_{E}(j)$ is the discrete value of the total load of a RDN, and $\varphi_{l j}=F_{L}(l) \cdot \Phi\left(P_{E}(j)\right)$ is the probability of the RDN load at the discrete value $L_{R D N}(l j)$. Assume a new set $L_{R D N}^{\prime}(m)$, which puts $L_{R D N}(l j)$ in ascending order, $L_{R D N}^{\prime}(m) \leq L_{R D N}^{\prime}(m+1), 0 \leq m \leq W \cdot n_{c}-1$. W $n_{c}$ is the number of discrete values of $L_{R D N}(m)$, the corresponding probability of RND load at value $L_{R D N}^{\prime}(m)$ is $\varphi_{m}$.

At a time instant, when $i=\varepsilon_{1}, m=\varepsilon_{2}\left(\varepsilon_{1}, \varepsilon_{2} \in N\right)$, voltage violation occurs, the probability of voltage violation in a RDN with SWTs and BEV charging loads, $P_{2}$, can be expressed by (8):

$$
P_{2}= \begin{cases}\sum_{m=\varepsilon_{c}}^{W \cdot n_{c}} \sum_{i=0}^{\varepsilon_{1}} F_{G}(i) \cdot \varphi_{m} & \text { when } V_{x}<V_{x \min } \\ \sum_{m=0} \sum_{i=\varepsilon_{1}}^{M} F_{G}(i) \cdot \varphi_{m} & \text { when } V_{x}>V_{x \max }\end{cases}
$$


where $\varepsilon_{1}, \varepsilon_{2} \in N, 0 \leq \varepsilon_{1} \leq M, 0 \leq \varepsilon_{2} \leq W \cdot n_{c}, x \in N$.

The formulation of voltage violation calculation in RDNs with SWTs and BEVs can be illustrated in Fig. 2. During the times when there are no BEVs plugged in and charging, the probability of voltage violation is determined by the probability distribution of the network load and SWT power production, as shown in Fig. 2 (a), and $W=7$ has been taken in the figure. When BEVs are connected into a RDN, the total load distribution can be expressed by $m$ discrete values shown in Fig. 2 (b), and the probability of voltage violation can then be determined by the probability distribution of the total RDN load and SWT power production. The probability of voltage violation of a distribution network equal to 0 is the ideal distribution network design. In practice, a realistic objective would be to reduce the probability of voltage violation to an acceptable level. $P_{1} \leq 0.05$ (or $P_{2} \leq 0.05$, at the times with BEVs) has been taken as the acceptable level in this paper, according to the requirement that the voltage must be maintained within the statutory limits for 95\% of a week in the standard EN-50160 [22].
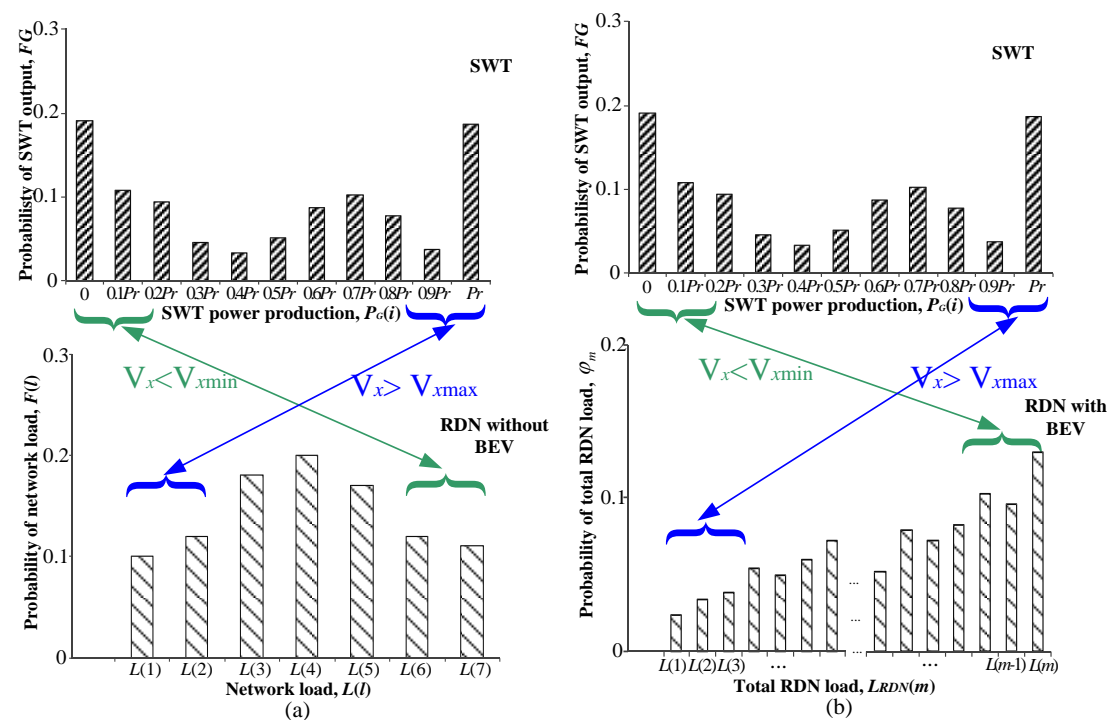

(b)

Fig. 2. Formulation of voltage violation calculation in RDNs with (a) SWTs and (b) SWTs and BEVs.

\section{Case Study}

\subsection{Example distribution network model}

The network shown in Fig. 3 represents a generic U.K. distribution network from a $33 \mathrm{kV}$ substation down to $400 \mathrm{~V}$ networks: based on data in [23]. The model is comprised of a $33 \mathrm{kV}$ three-phase grid supply point and six $11 \mathrm{kV}$ outgoing feeders: each $11 \mathrm{kV}$ feeder supplies eight 11/0.4 kV $500 \mathrm{kVA}$ distribution transformers. Five feeders are modeled as lumped loads, whilst the sixth feeder is considered in more detail, i.e. one of the eight $400 \mathrm{~V}$ radial distribution lines on the sixth feeder is modified to represent a RDN. In the model used 384 homes are distributed in the RDN. One of the feeder cables in the RDN is represented in detail, with each L representing 12 homes. Balanced three-phase loads, with a power factor 0.98 , are spaced uniformly along the feeder cables. SWTs rated at $3.5 \mathrm{kVA}$ with a power factor 0.95 and BEVs are to be integrated into these homes.

\subsection{Penetration levels of SWTs and BEVs}

This paper represents penetration level of SWTs as the total installed SWT capacity in relation to the peak load of the RDN, e.g. when 32 homes in the RDN have SWTs installed, the penetration level of SWTs is $22.4 \%$. This paper considers two SWT penetration levels, $22.4 \%$ and $44.8 \%$, i.e. 1 out of 12 homes and 2 out of 12, respectively, have an SWT installed, and these could be reasonable penetrations in the future network [1]. 


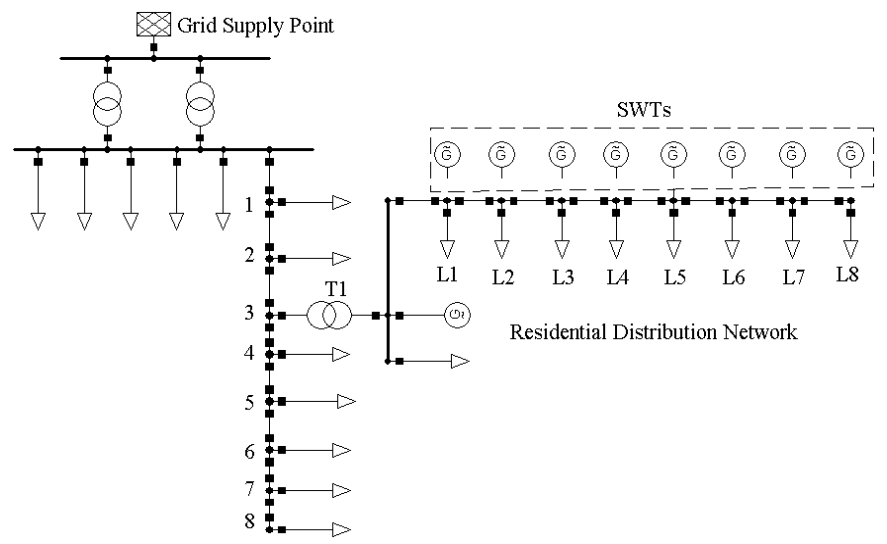

Fig. 3. Example U.K. distribution network with SWTs.

The penetration of BEVs in this paper refers to the ratio of BEVs to the total number of vehicles in the RDN. According to the analysis in [18], the ratio of the number of cars in the RDN to the total number of vehicles in the UK is assumed to be the same as the proportion of the RDN electricity usage to the UK's peak load, i.e. 288 cars are possessed by residents in the RDN (75\% of homes on average have a car). In [25], $7.07 \%$ and $48.56 \%$ penetration levels of EVs were referred to as the low and high EV-uptake levels in 2030 in the UK. This paper considers two penetration levels of BEV, $22.2 \%$ and 44.4\%, i.e. 64 and 128 of the 288 cars in the RDN are BEVs.

Four scenarios of SWT and BEV penetrations have been considered, as shown in Table 1.

Table 1. Simulation scenarios

\begin{tabular}{ccc}
\hline \hline \multirow{2}{*}{ Scenario } & \multicolumn{2}{c}{ Penetration levels } \\
\cline { 2 - 3 } & SWT & BEV \\
\hline Scenario 1 & $22.4 \%$ & $0 \%$ \\
Scenario 2 & $44.8 \%$ & $0 \%$ \\
Scenario 3 & $44.8 \%$ & $22.2 \%$ \\
Scenario 4 & $44.8 \%$ & $44.4 \%$ \\
\hline \hline
\end{tabular}

Table 2. Voltage violation probability comparison by statistical time series \& improved statistical time series method

\begin{tabular}{|c|c|c|c|c|c|c|c|c|c|c|c|}
\hline \multirow{3}{*}{ Scenario } & \multirow{3}{*}{ Time of day } & \multicolumn{5}{|c|}{ Weekdays } & \multicolumn{5}{|c|}{ Weekends } \\
\hline & & \multicolumn{3}{|c|}{ Probability } & \multicolumn{2}{|c|}{ Difference, $\%$} & \multicolumn{3}{|c|}{ Probability } & \multicolumn{2}{|c|}{ Difference, $\%$} \\
\hline & & $T S$ & STS & ISTS & STS & ISTS & $T S$ & STS & ISTS & STS & ISTS \\
\hline \multirow{4}{*}{ Scenario 1} & $1: 00$ & 0.168 & 0.144 & 0.161 & -14.3 & -4.2 & 0.182 & 0.151 & 0.195 & -17.0 & 7.1 \\
\hline & 11:00 & 0.02 & 0 & 0.019 & -100.0 & -5.0 & 0.02 & 0 & 0.010 & -100.0 & -50.0 \\
\hline & $18: 30$ & 0 & 0 & 0 & - & - & 0 & 0 & 0 & - & - \\
\hline & 22:00 & 0 & 0 & 0 & - & - & 0 & 0 & 0 & - & - \\
\hline \multirow{4}{*}{ Scenario 2} & $1: 00$ & 0.286 & 0.254 & 0.303 & -11.2 & 5.9 & 0.292 & 0.260 & 0.324 & -11.0 & 11.0 \\
\hline & 11:00 & 0.165 & 0.178 & 0.177 & 7.9 & 7.3 & 0.12 & 0.117 & 0.131 & -2.5 & 9.2 \\
\hline & $18: 30$ & 0.08 & 0 & 0.007 & -100.0 & -91.3 & 0.08 & 0 & 0.006 & -100.0 & -92.5 \\
\hline & 22:00 & 0.12 & 0 & 0.015 & -100.0 & -87.5 & 1.10 & 0 & 0.07 & -100.0 & -93.6 \\
\hline
\end{tabular}

“TS” denotes Times Series method, "STS" Statistical Time Series method, "ISTS” Improved Statistical Time Series method

\section{Simulation Results and Discussions}

Load data from 2004 [24] and wind speed data for Camborne, UK for the same year [26] are used in this paper. The daily probabilities of voltage violation of the RDN under scenario 1 and 2, where only SWT penetration is considered, are shown in Fig. 4. When considering penetration by both SWT and $\mathrm{BEV}$, the daily probabilities of voltage violation for scenarios 3 and 4 are shown in Fig 5, scenario 2 has been included for comparison. Note that energy consumption in week days tend to have the same profile, days in a weekend have a different profile, hence the presentation of the data in two graphs. 
Fig. 4 illustrates that (i) voltage violation is more likely to happen in the early hours of a day in a RDN, and (ii) the voltage rise caused by the power injection of SWTs is the main reason for voltage violation.

The results obtained by the statistical time series method and the improved method for scenarios 1 and 2 are compared with those from the Time Series (TS) method in Table 2. Probabilities of voltage violation at $01: 00,11: 00,18: 30$ and 22:00, when network loads represent $25 \%, 50 \%, 100 \%$ and $75 \%$ of the daily peak load respectively, are shown.

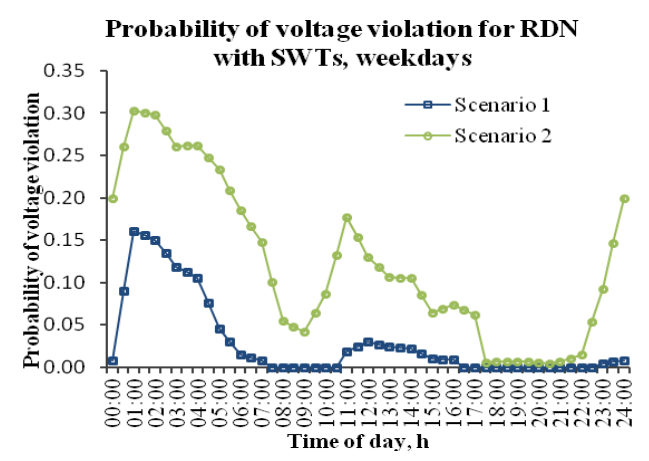

(a)

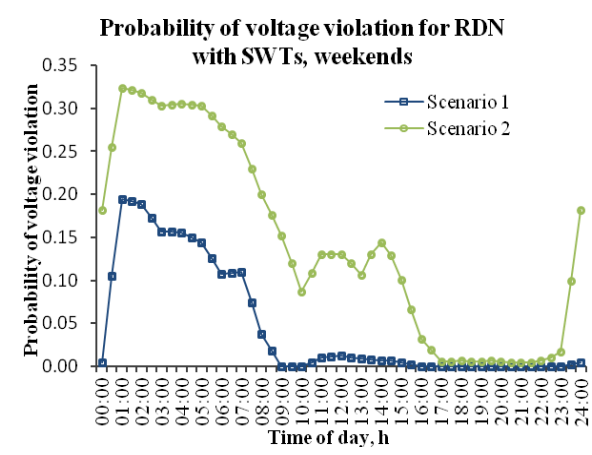

(b)

Fig. 4. Probabilities of voltage violation of the RDN with SWTs for (a) weekdays and (b) weekends.

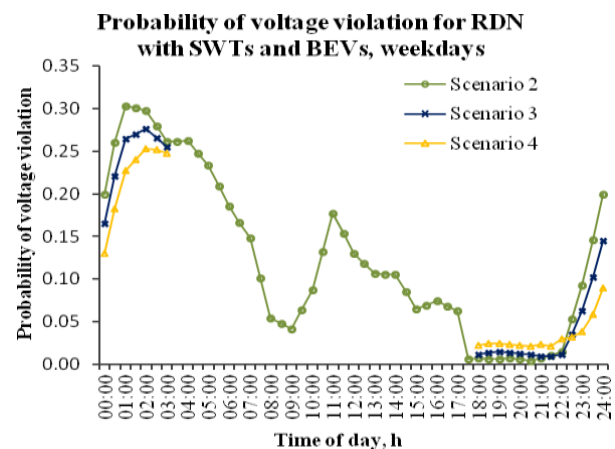

(a)

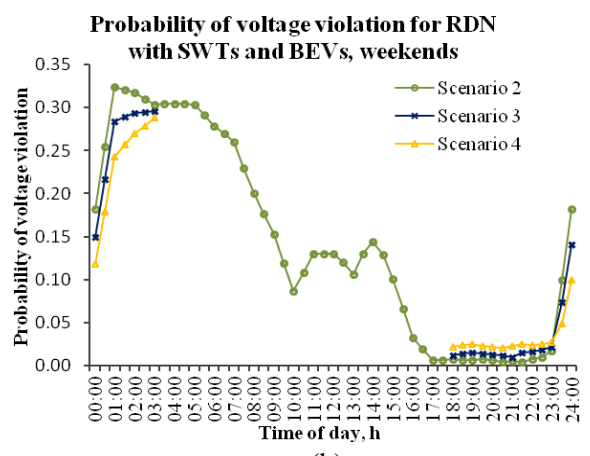

(b)

Fig. 5 Probabilities of voltage violation of the RDN with SWTs and BEVs for (a) weekdays and (b) weekends.

Taking the results of the TS approach as reference, Table 2 demonstrates that the improved statistical time series (ISTS) method is closer to the output of the TS results than the statistical time series (STS). When using the ISTS method, the load data statistics take into account the extreme load conditions e.g. coincidental peak load. Voltage violation is more likely to happen under these load conditions, thus the voltage violation simulation by the proposed method can be more accurate than using statistical time series approach.

\section{Conclusions}

An improved statistical time series method is presented to evaluate the voltage violation of RDNs with different penetration levels of SWTs and BEVs. In addition to consideration of time-variations in wind speeds and BEV charging in the statistical time series approach, the improved method models the variations in system load caused by the randomness of load switching by customers. The extreme load conditions which could cause a voltage violation are considered in the voltage violation quantification of RDNs in the improved method and, as a result, studies using this method can provide more accurate results of daily probability distribution of voltage violation of a RDN than those using the statistical time series approach. 


\section{References}

[1] RenewableUK. (Apr. 5, 2011). Small wind systems UK market report 2011. [Online]. Available: http://windcrop.co.uk/wpcontent/uploads/2012/12/Small_Wind_Systems_Market_Report_20111.pdf

[2] Qian K, Zhou C, Allan M, Yuan Y. Modeling of load demand due to BEV battery charging in distribution systems. IEEE Transactions on Power Systems, May 2011; 26(2):802-810.

[3] Masters CL. Voltage rise - the big issue when connecting embedded generation to long $11 \mathrm{kV}$ overhead lines. Power Engineering Journal, Feb. 2002; 16(1):5-12.

[4] Keane A, O'Malley M. Optimal allocation of embedded generation on distribution networks. IEEE Transactions on Power Systems, Aug. 2005: 20(3):1640-1646.

[5] Clement-Nyns K, Haesen E, Driesen J. The impact of charging plug-in hybrid electric vehicles on a residential distribution grid. IEEE Transactions on Power Systems, Feb. 2010; 25(1):371-380.

[6] Recommendations for the Connection of Small-scale Embedded Generators (up to 16 A per phase) in Parallel with Public Low-voltage Distribution Networks, Engineering Recommendation. G83/1, Sep. 2003.

[7] Boehme T, Wallace AR, Harrison GP. Applying time series to power flow analysis in networks with high wind penetration. IEEE Transactions on Power Systems, Aug. 2007; 22(3):951-957.

[8] Ochoa LF, Padilha-Feltrin A, Harrison GP. Time-series based maximization of distributed wind power generation integration. IEEE Transactions on Energy Conversion, Sep. 2008; 23(3):968-974.

[9] Ochoa LF, Dent CJ, Harrison GP. Distribution network capacity assessment: variable DG and active networks. IEEE Transactions on Power Systems, Feb. 2010; 25(1):87-95.

[10] Thomson M, Infield DG. Network power-flow analysis for a high penetration of distributed generation. IEEE Transactions on Power Systems, Aug. 2007; 22(3):1157-1162.

[11] Persaud S et al., Impact of remotely connected wind turbines on steady state operation of radial distribution networks. IEE Proceedings Generation Transmission \& Distribution, May 2000; 147(3):157-163.

[12] Trichakis P et al., Predicting the technical impacts of high levels of small-scale embedded generators on low-voltage networks. IET Renewable Power Generation, Dec. 2008; 2(4): 249-262.

[13] Ayres HM. Method for determining the maximum allowable penetration level of distributed generation without steady-state voltage violations. IET Generation Transmission \& Distribution., Apr. 2010; 4(4):495-508.

[14] Zhang S et al., Statistical voltage quality assessment method for grids with wind power generation. IET Renewable Power Generation, Jan. 2010; 4(1):43-54.

[15] Masters CL, Mutale J, Strbac G, Curcic S, Jenkins N. Statistical evaluation of voltages in distribution systems with embedded wind generation. IEE Proceedings Generation Transmission \& Distribution, Jul. 2000; 147(4):207-212.

[16] Villafafila R et al., Probabilistic assessment of wind power production on voltage profile in distribution networks. In: Proc. of Int. Conf. Electrical Power Quality and Utilization, 2007:1-5.

[17] Demailly F et al., Numerical tools and models for Monte Carlo studies of the influence on embedded generation on voltage limits in LV grids. Jul. 2005; 20(3):2343-2350.

[18] Long C, Farrag MEA, Zhou C, Hepburn DM. Statistical quantification of voltage violations in distribution networks penetrated by small wind turbines and battery electric vehicles. IEEE Transactions on Power Systems, 2013, 28(3)2403-2411.

[19] Lun IYF, Lam, JC. A study of Weibull parameters uses long-term wind observations. Renewable Energy, Jun. 2000; 20(2):145-153.

[20] Global Business Intelligence Research. (Jan. 2010) Future of global advanced batteries market outlook to 2020: opportunity analysis in electronics and transportation. [Online]. Available: http://www.reportlinker.com/p0174566/Future-of-GlobalAdvanced-Batteries-Market-Outlook-to-Opportunity-Analysis-in-Electronics-and-Transportation.html

[21] Madrid C et al., Performance Characterization-1999 Nissan Altra-EV with Lithium-Ion Battery. Southern California EDISON, Sep. 1999.

[22] British Standard on Voltage Characteristics of Electricity Supplied by Public Distribution Systems, BS-EN-50160, 1995.

[23] Ingram $\mathrm{S}$ et al., The impact of small scale embedded generation on the operating parameters of distribution networks. Department of Trade and Industry, London, U.K., Jun. 2003.

[24] DTI Centre for Distributed Generation and Sustainable Electrical Energy. (2006, Mar.). United Kingdom generic distribution system phase one. [online]. Available: http://www.sedg.ac.uk/ukgds.htm.

[25] Papadopoulos P et al., Electricity demand with electric cars in 2030: comparing Great Britain and Spain. Journal of Power and Energy, Aug. 2011; 225(5):551-566.

[26] UK Meteorological Office. (2011). Met Office Wind Profiler data (1998-onwards) [online]. Available: http://badc.nerc.ac.uk/view/badc.nerc.ac.uk_ATOM_dataent_ukmowindpr. 